\title{
Bacterial evolution and the Bak-Sneppen model
}

\author{
Indrani Bose and Indranath Chaudhuri ${ }^{\ddagger}$ \\ Department of Physics, \\ Bose Institute, \\ 93/1, Acharya Prafulla Chandra Road, \\ Calcutta-700 009, India. \\ $\ddagger$ Department of Physics, \\ St. Xavier's College, \\ 30, Park Street, Calcutta-700016, India.
}

\begin{abstract}
Recently, Lenski et al [1, 2, 3] have carried out several experiments on bacterial evolution. Their findings support the theory of punctuated equilibrium in biological evolution. They have further quantified the relative contributions of adaptation, chance and history to bacterial evolution. In this Brief Report, we show that a modified M-trait Bak-Sneppen model can explain many of the experimental results in a qualitative manner.
\end{abstract}

bf PACS : 87.23.Kg, 05.65.+b,87.17.Ee

Recently, a set of experiments has been carried out on bacterial evolution which has given rise to a new sub-discipline in the area of evolutionary biology, namely, experimental evolution [1, 2, 3, 4]. This implies the study, in the laboratory, of the fundamental processes of evolutionary change, namely, spontaneous mutation and adaptation by natural selection. Experiments on evolutionary dynamics require passage through thousands of generations which is an impossibility for most living species. Microorganisms like bacteria, yeast or viruses, however, have very short generation times (bacteria like E.coli have about seven generations everyday in sugar solution). The short generation times make it possible to observe population dynamics over thousands of generations and thereby address a wide range of evolutionary questions. Darwin laid the foundation of evolutionary biology by setting forth the principle of adaptation by species through natural selection [5]. It is now known that spontaneous mutation plays an important role in generating differences among individual organisms. A mutation involves a change 
in the base sequence of a DNA and can occur with a certain probability during cell division. Mutations are random events and may be harmful, neutral or beneficial as regards their effect on an organism. According to the modern version of Darwin's theory, random mutations give rise to heritable differences among organisms whereas natural selection tends to increase the number of fitter variants. The processes of reproduction, mutation and natural selection are responsible for evolution of species or that of a single population.

In this Letter, we consider a set of experiments carried out by Lenski and co-workers [1], 2, 3] on the bacteria E.coli. As pointed out by them [6], a large population size of E.coli ensures that a large number of mutations occur in every generation so that the origin and the fate of genetic variation can be well studied. It is possible to store bacteria in suspended animation at low temperatures. One can then measure the relative fitness (RF) of descendant and ancestral populations by placing them in direct competition. The RF is expressed as the ratio of the realized growth rates of the two populations. Finally, the populations are easy to handle and propagate so that intensive replication of experiments is possible allowing subtle effects to be measured. In the first experiment [1, 2], Lenski and co-workers studied an evolving bacterial population for approximately 10,000 generations. The bacterial population was allowed to expand to $5 \times 10^{8}$ cells in low sugar solution. At the end of the growth period (one day), 1/100 th of the population was siphoned into a fresh flask of food to allow the population to evolve. Since there was a 100-fold expansion of the bacterial population in a day, 6.6 $\left(=\log _{2} 100\right)$ generations of binary fussion occurred during this time. Every fifteen days, a sample of the population was frozen for later analysis giving rise to a 'frozen fossil record'. Since the population originated from a single cell, mutations, about $10^{6}$ everyday, provided the sole source of genetic variation. Four years later, Lenski et al had data for the evolving bacterial population over 10,000 generations. They measured two quantities, the average cell size which is preserved in fossil records, and the RF. They found that the average cell size and the RF grow in a punctuated manner, i.e., in steps, as a function of time (number of generations) when the data are plotted every 100 generations (inset of Fig.1). At a larger interval of 500 generations, the changes appear to be gradual (inset of Fig.2 ). A major debate in evolutionary biology revolves around the question of whether evolution is best described as a gradual change or occurs in bursts. In the latter 
case, short periods of evolutionary activity are punctuated by long periods of stasis. This is the theory of punctuated equilibrium (PE). Lenski et al's data seem to support this theory though their interpretation is open to controversy [7]. The experiment, however, clearly demonstrates that both the average cell size and the RF of the evolving population grow over a certain time interval. In the first 2000 generations or so, there is a rapid growth followed by a period of slower growth till the growth is imperceptible. The bacteria, being in low sugar solution, have to compete for the food. Natural selection favours the mutations that confer some competitive advantage in exploiting the experimental environment. This leads to adaptation of the bacterial population to the environment through the emergence of larger and fitter varients.

In the second experiment [2], twelve populations of E.coli were evolved over 10,000 generations in identical environments. Each population was founded by a single cell from an asexual clone to eliminate genetic variation within and between populations. It was found that the replicate populations, after 10,000 generations, differ considerably from one another in both the average cell size and the RF (inset of Fig.3 ), even though the populations evolved in identical environments. In the third experiment [3], the relative contributions of adaptation, chance (mutations) and history to evolution were investigated. Twelve replicate populations were founded from a single clone of E.coli and serially propagated for 2000 generations in glucoselimited medium. The 12 populations had similar fitness values when evolving in glucose medium but when put in a maltose-limited medium showed large differences in fitness values. Some populations thrived while some others were found to languish. Ancestral fitness values of the populations in maltose were thus very heterogeneous (inset of Fig.4). One genotype from each of the 12 replicate populations was cloned and from this 3 new replicate populations were founded. The 36 populations were then evolved under ancestral conditions in the maltose medium. The inset in Fig. 4 shows the derived fitness in maltose the versus ancestrial fitness in maltose for 36 populations.

Bak and Sneppen (BS) 88, 9] have proposed a model to study the evolution of interacting species. The model self-organises into a critical state characterised by power-laws of various types. Further, evolutionary activity in this state exhibits PE. Boettcher and Paczuski [10] have proposed the Mtrait BS model in which each biological species is characterised by M traits, instead of one trait (fitness) as in the original BS model. The major fo- 
cus of these studies has been the self-organised critical (SOC) state and its various features. The temporal evolution leading to the SOC state has not been studied in detail. In this letter, we show that, with some simple modifications of the $\mathrm{M}=2 \mathrm{BS}$ model, many of the experimental results of Lenski et al can be reproduced in a qualitative manner. Our study highlights the significance of the dynamics in the BS-type models before the SOC state is reached. The BS model gives a coarse-grained representation of real evolution but contains the essential elements to capture the course of evolution. In our modified $\mathrm{M}=2 \mathrm{BS}$ model, the bacterial population is divided into $\mathrm{N}$ categories. Each category contains bacteria of similar characteristics. The N categories correspond to the $\mathrm{N}$ sites of a one-dimensional (1D) lattice with periodic boundary conditions. In the original BS model, each site represents a species. Two traits, namely, cell size and fitness are associated with the population at each site $\mathrm{i}, \mathrm{i}=1,2, \ldots, \mathrm{N}$. One assigns a number (between 0 and 1) to each of the traits at all the $\mathrm{N}$ sites. At each time step, the two sites with the minimum values for each of the two traits are identified. Mutations occur to bring about changes in the traits. The minimum random numbers are replaced by new random numbers. In the original $\mathrm{M}=2 \mathrm{BS}$ model, the minimum value, amongst all the $2 \mathrm{~N}$ values of the two traits, is replaced by a new random number. The random numbers associated with any one of the traits of the neighbouring sites are also replaced by new random numbers. This is to take into account the linkage of neighbouring populations in food chain. The last two steps are repeated and averages are taken for both the traits locally (over 40 sites) and globally (over 2000 sites). Unlike in the original BS model, we calculate quantities from the very beginning and not after the SOC state is reached. The SOC state corresponds to the region in which evolutionary growth is imperceptible and fluctuates around an average value. Fig. 1 shows the variation of the RF versus time. The inset shows the experimental data [1, 2]. The averages are taken over 40 sites and every 100 time steps. The local averaging gives rise to an improved quality of data points. Fig. 2 shows the corresponding variation with averages taken every 500 time steps and over the whole lattice. For a very large lattice one needs to take only global averages. The RF is defined to be the ratio of the current fitness and the initial fitness at time $t=0$. In the actual experiment, fitness is related to the growth rate of the bacterial population via replication. The $\mathrm{RF}$ increases rapidly during the first 2000 generations. After that the growth becomes slower till it becomes imperceptible. The rapid growths can be fit 
by an hyperbolic model

$$
y=x_{0}+\frac{a x}{b+x}
$$

for both the experimental and simulation data. During the periods of punctuation the beneficial mutations are few with no significant effect. When such mutations occur in quick succession, rapid evolutionary growth is observed. Recent research findings [11] have highlighted the importance of large beneficial mutations in the initial stages of evolutionary growth. Organisms must adapt to the new conditions fairly quickly in order to survive. Later, mutations with smaller effect fine-tune the adaptation. Fig.2 shows this clearly with a rapid evolutionary growth in the first 2000 generations brought about by beneficial mutations of large effect. The growths are imperceptible when the bacterial population gets adapted to its environment. The average cell size as a function of time has similar variations as in the case of the RF (Figs. 1 and 2).

Fig.3 shows a comparison of the simulation data for the RF with the data (inset) of the second experiment of Lenski et al [2]. The plots show that the independent populations diverge significantly from one another . In the simulation, the initial random number seed was chosen to be different for the six populations. The average fitness at time $t=0$ does not vary noticably from one population to another. Fig.4 shows the simulation results for the third experiment [3] with $2 \times 4$ populations rather than the $3 \times 12$ populations in the actual experiment. In the experiment, the populations growing in glucose- limited medium were transferred after 2000 generations to maltose-limited medium. In the latter medium, the average fitness values of the populations showed large differences. Thus, in the simulation for the maltose medium, one starts with widely different average fitness values for the populations. The populations are evolved for 1000 time steps. One finds, in agreement with the experimental results, that after 1000 generations, the average fitness values have similar magnitudes. This shows that adaptation and chance (effect of mutation) have eliminated the initial heterogeneity in average fitness values to a great extent. The effect of history (initial heterogeneity) is reduced after several generations of evolution. The effect of adaptation is pronounced (shown by the evolution of the data points above the isocline). The effect of chance is seen in the small dispersion in the average fitness values of the two populations corresponding to each genotype. Lenski 
et al [3] have given quantitative estimates of the relative contributions of adaptation, chance and history to average fitness and cell size before and after 1000 generations in maltose.

To conclude, we have described a set of experiments by Lenski et al on bacterial evolution and have shown that a $\mathrm{M}=2$ BS-type model gives a satisfactory description of the experimental data. Both simulation and experiments support the theory of PE in bacterial evolution. Recent exhaustive studies of fossil beds lend support to this theory [12]. The model is applied to evolving bacterial populations rather than to interacting species as in the original BS model. Also, as emphasized earlier, the BS-type models have so far been studied in the context of self-organised criticality. In this Brief Report, we highlight the significance of the temporal evolution of BS-type models before the self-organised critical state is reached. This temporal evolution needs to be characterised in greater detail to give a quantitative fit to the experimental data of Lenski et al. For example, appropriate change in random number intervals may be necessary for a good fit. Lenski et al [13], 14, 15, 16] have developed theories based on standard population-genetics approaches to explain some of their experimental data. The BS-type model gives a coarse-grained description to bacterial evolution but, as shown in this Letter, it captures the major features of the evolutionary growth of bacterial populations. Experiments have recently been performed on the growth of RNA virus fitness [17]. The adaptive evolutionary capacity in this case is overwhelming. The gain in fitness is nearly 5000 after 50 passages. In that time, the gain in E.coli fitness changes can be explained by an hyperbolic model whereas RNA virus evolution follows exponential kinetics. The RNA virus evolution follows exponential kinetics whereas E.coli evolution corresponds to hyperbolic growth. A BS-type model can explain the hyperbolic growth but not the exponential one. For bacterial populations, Lenski et al's remarkable experiments open up possibilities of a rich interplay between theory and experiments. The self-organised critical state in the experiment and simulation corresponds to the region in which growth is imperceptible. With appropriate designing of experiments, the phenomenon of self-organised criticality in an evolving bacterial population can be studied experimentally. 


\section{Acknowledgement}

We are greatful to R.E.Lenski for sending us his publications on bacterial evolution.We also acknowledge the help from the Distributed Informatics Centre, Bose Institute, in preparing the manuscript. 


\section{Figure Captions}

Fig.1 Relative fitness versus time in experiment [2] and simulation. A local average is taken over 40 sites in simulation. The experimental data points are taken every 100 generations.

Fig.2 Relative fitness versus time in simulation and experiment (inset) [2]. The data points are taken every 500 generations and the average is over the 2000 sites of the lattice.

Fig.3 Trajectories for relative fitness in six replicate populations of bacteria during 10,000 generations (simulation) and the same for twelve replicate populations (experiment). The data points are taken at an interval of 500 generations.

Fig.4 Evolution of fitness during 1000 generations in maltose. Derived versus ancestral values for relative fitness in 8 populations (simulation) and the same for 36 populations (experiment). The different symbols indicate the different progenitor genotypes. 


\section{References}

[1] S.F.Elena, V.S.Cooper and R.E.Lenski, Science 272, 1802(1996)

[2] R.E.Lenski and M.Travisano, Proc. Natl. Acad. Sci. 91, 6808 (1994)

[3] M.Travisano, J.A.Mongold, A.F.Bennett and R.E.Lenski, Science 267, $87(1995)$

[4] R.E.Lenski in Encyclopedia of Microbilogy, Volume 2 ed. by J.Lederberg, page 125 (Academic Press, 1992)

[5] C.Darwin, The Origin of Species by Means of Natural Selection (Murray, London,1859 : reprinted Penguin, New York, 1976)

[6] M.Travisano, F.Vasi and R.E.Lenski, Evolution 49, 189 (1995)

[7] J.A.Coyne and B.Charleswork, Science 274, 1748 (1996), S.F.Elena, V.S.cooper and R.E. Lenski, Science 274, 1749 (1996)

[8] P.Bak and K.Sneppen, Phys. Rev. Lett. 71, 4083 (1993)

[9] P.Bak, How Nature Works: The Science of Self-Organised Criticality, (Springer, New York)

[10] S.Boettcher and M.Paczuski, Phys.Rev.Lett. 76, 348 (1996)

[11] V.M, Science 284, 2106 (1999)

[12] R.A.Kerr, Science 267, 1421 (1995)

[13] R.E.Lenski, M.R.Rose, S.C.Simpson and S.C.Tadler, Am. Nat. 138, 1315 (1991)

[14] P.J.Gerrish and R.E.Lenski, Genetica 102/103, 127 (1998)

[15] P.A.Johnson, R.E.Lenski and F.C.Hoppensteadt, Proc. R. Soc. Lond. B 259, 125 (1995)

[16] F.Vasi, M.Travisano and R.E.Lenski, Am. Nat. 144, 432 (1994) 
[17] I.S.Novella, E.A.Duarte, S.F.Elena, A.Moya, E.Domingo and J.H.Holland, PNAS 92, 5841 (1995), L.S.Tsimring, H.Levine and D.A.Kessler, Phys. Rev. Lett. 76, 4440 (1996) 


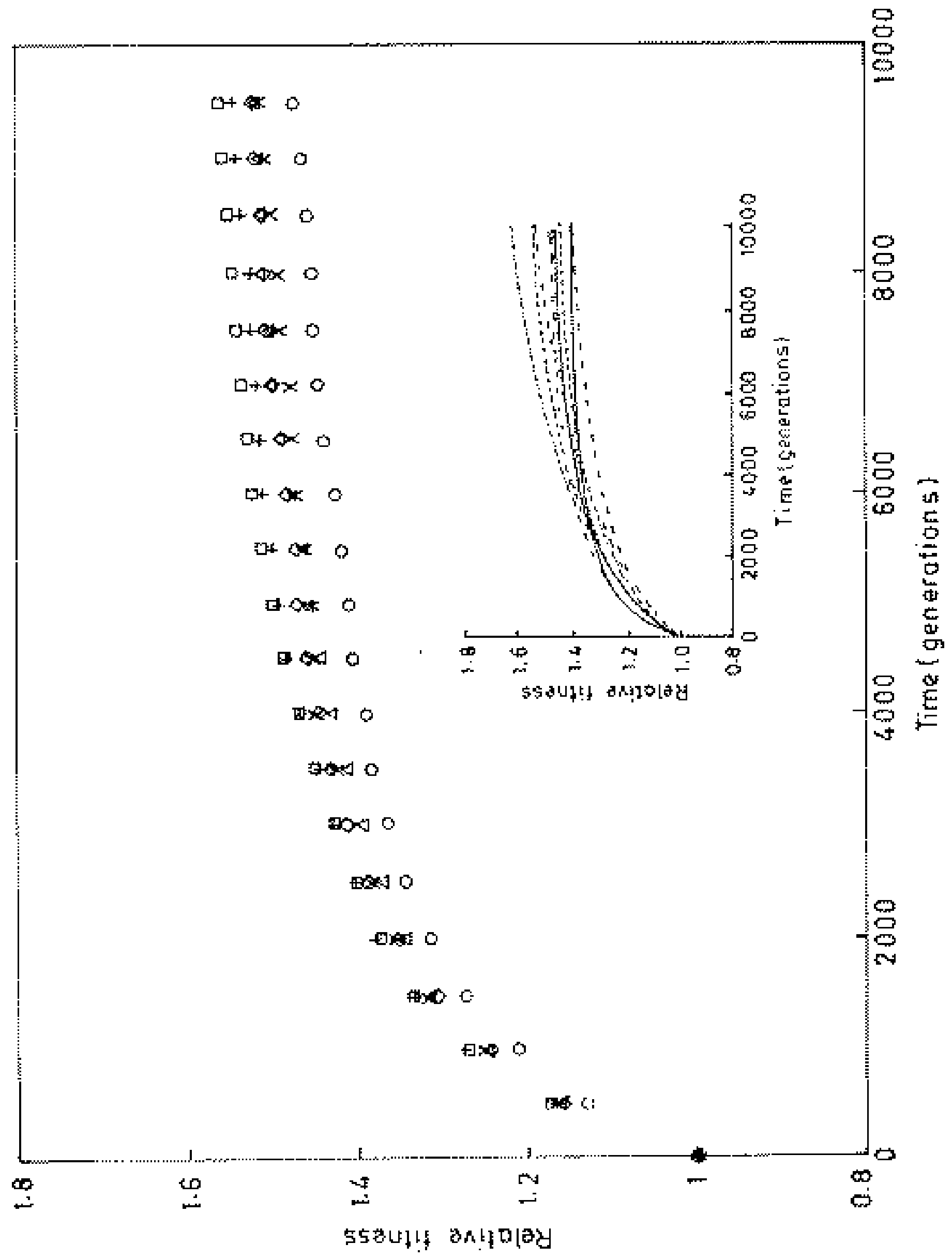




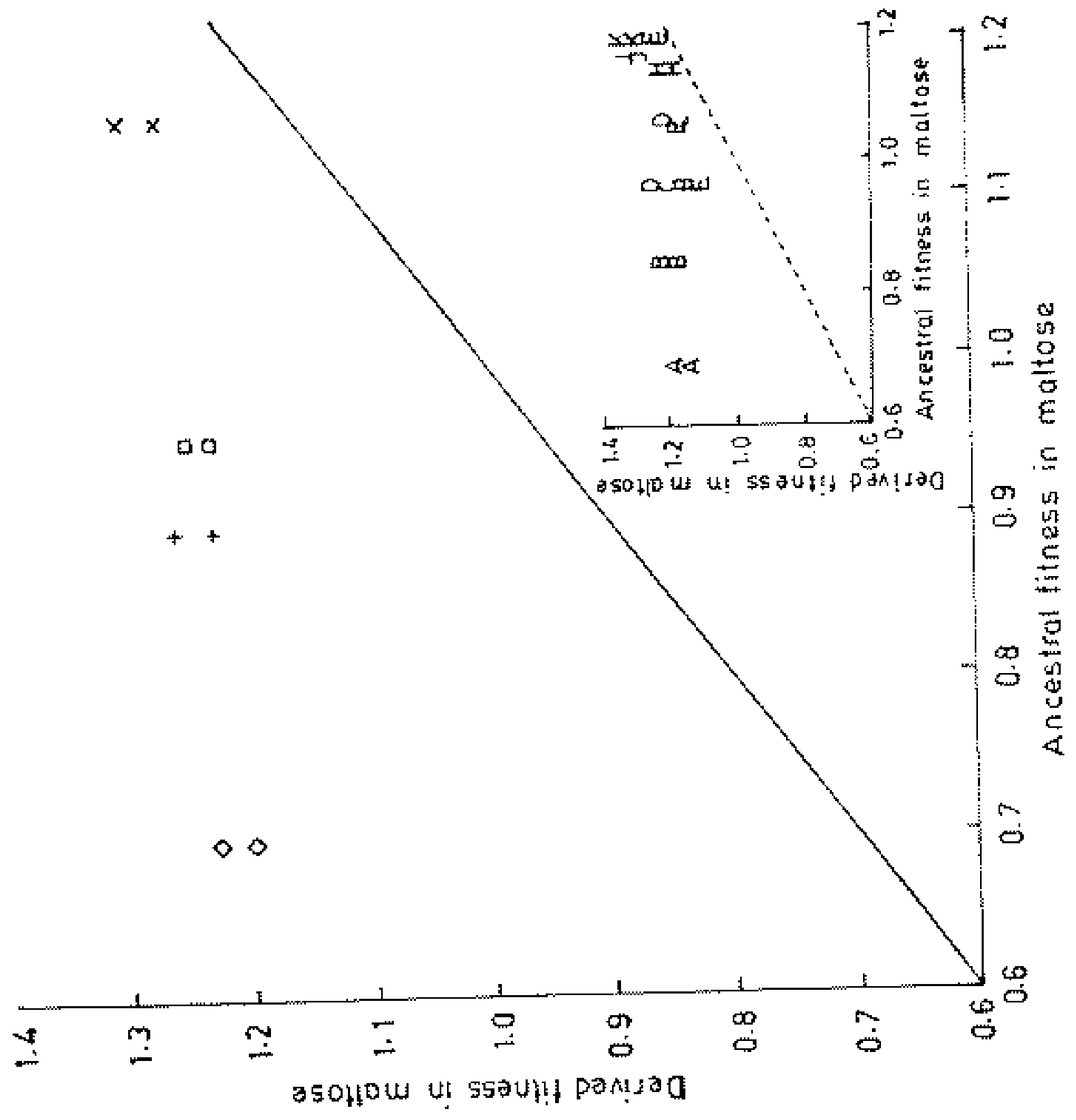




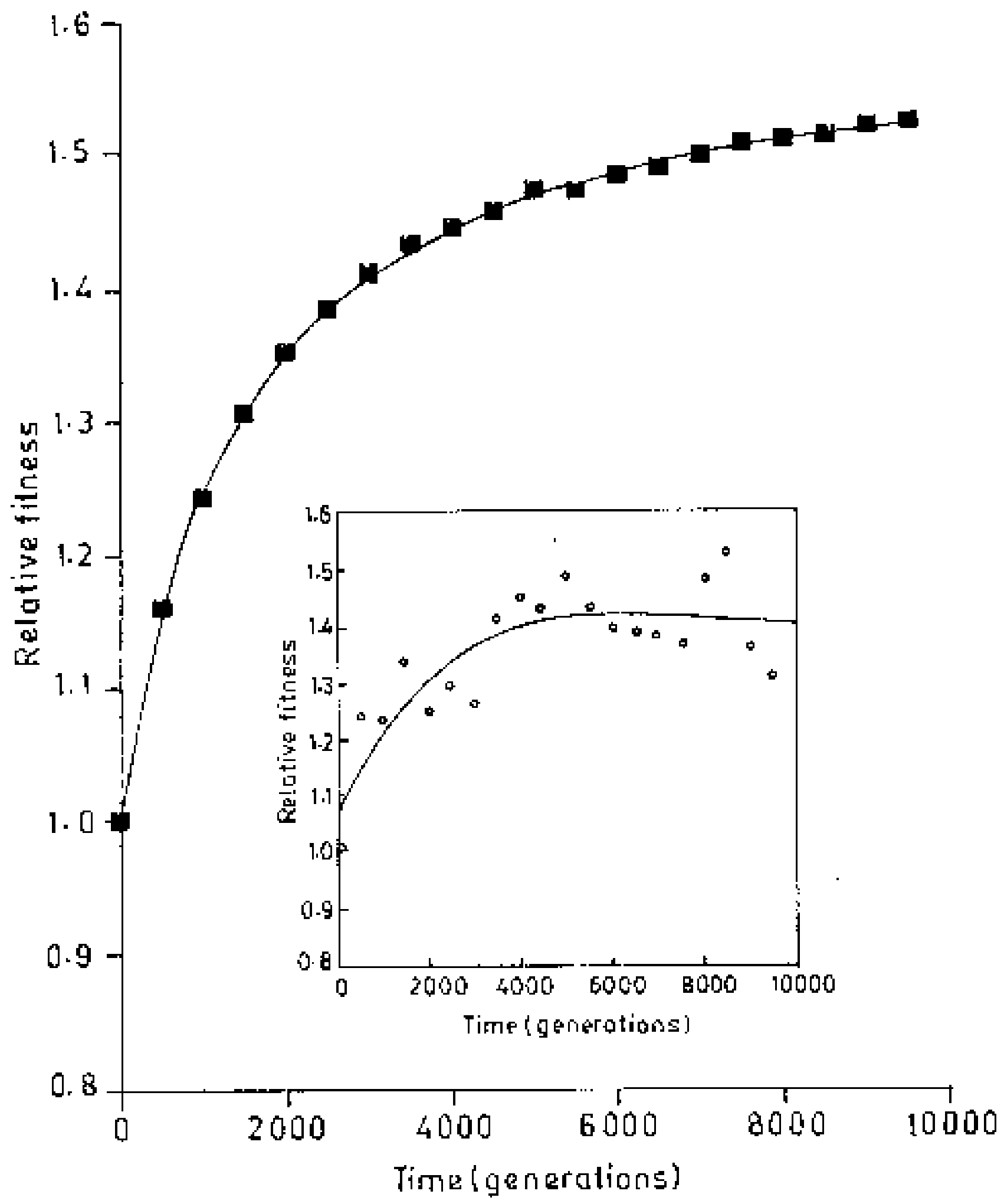




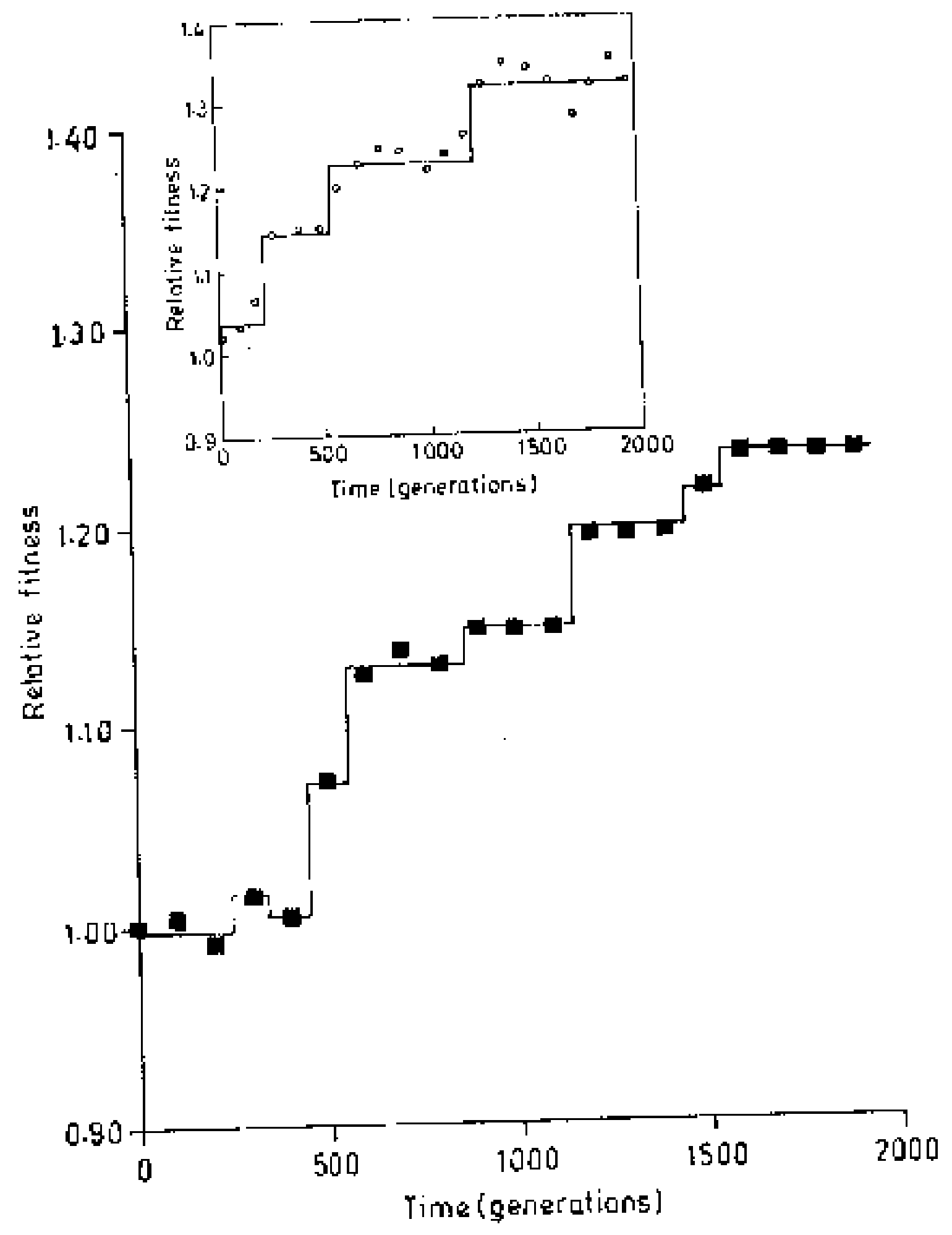

